\title{
Pesquisa multicêntrica de indicadores clínicos para a detecção precoce de riscos no desenvolvimento infantil
}

\section{Resumo}

A partir da teoria psicanalítica, foram desenvolvidos Indicadores clínicos de risco para o desenvolvimento infantil (IRDIs) observáveis nos primeiros 18 meses de vida da criança. O pressuposto é que esses indicadores clínicos (IRDIs) podem ser empregados pelos pediatras durante a consulta nas unidades básicas e/ou centros de saúde e podem ser úteis para detectar precocemente transtornos psíquicos do desenvolvimento infantil. Os objetivos do estudo são: 1) descrever o perfil epidemiológico dos IRDIs; 2) verificar sua capacidade de predição para transtornos psíquicos na infância; 3) estabelecer indicadores de desenvolvimento psíquico para complementação da ficha de desenvolvimento proposta pelo Ministério da Saúde para o acompanhamento do desenvolvimento de crianças de 0 a 5 anos; 4) verificar sua associação com características clínicas e demográficas.

O estudo utilizará um desenho de corte transversal seguido por estudo longitudinal numa amostra de crianças, nas 
faixas etárias de 0-3 meses e 29 dias; 4-7 meses e 29 dias; 8-11 meses e 29 dias e 12-18 meses, atendidas na clínica pediátrica nas unidades e/ou centros de saúde em dez cidades brasileiras (totalizando 12 centros). Os IRDIs serão utilizados por pediatras treinados durante a consulta clínica regular no período de 18 meses. A análise dos dados da primeira etapa do estudo será composta de uma descrição epidemiológica para estimar a sua associação com variáveis clínicas e epidemiológicas. Após três anos de seguimento, as crianças serão avaliadas para identificação de transtornos psicológicos ou psiquiátricos e verificadas as associações com os IRDIs.

\section{Introdução e justificativa}

A presente pesquisa parte da concepção psicanalítica do desenvolvimento psíquico da criança de 0 a 36 meses. Visa à elaboração de indicadores clínicos para a detecção precoce de transtornos psíquicos do desenvolvimento infantil, com fins preventivos, bem como ao aperfeiçoamento de pediatras para atuar como agentes dessa detecção e prevenção.

O pressuposto que norteia esta pesquisa é o de que as bases da saúde mental se estabelecem nos primeiros anos de vida e são dependentes das relações corporais, afetivas e simbólicas que se estabelecem entre o bebê e sua mãe (ou substituto). Essas relações promovem a inserção do ser humano na cultura e constroem uma subjetividade, eixo organizador do desenvolvimento em todas as suas vertentes. Falhas nesse processo de constituição da subjetividade ocasionam transtornos psíquicos do desenvolvimento infantil.

Assim, a proposição da presente pesquisa justifica-se:

a) pelo impacto epidemiológico que os transtornos psíquicos de desenvolvimento na infância produzem;

b) pelas dificuldades que apresentam os pediatras na detecção desses transtornos;

c) pela evidência de que a deteç̧ão precoce produz uma mudança significativa no desfecho clínico da criança;

d) pela insuficiência das escalas atualmente existentes em relação aos aspectos psíquicos do desenvolvimento.

\section{Impacto epidemiológico}

Historicamente, os serviços públicos voltados para os transtornos mentais do desenvolvimento na infância se mostraram inadequados ou inexistentes. Isto se deve, entre outras razões, ao fato de que os parcos recursos do setor da saúde 
foram utilizados predominantemente para combater as causas da mortalidade e morbidade infantis, como a diarréia, as doenças imunopreveníveis, a desnutrição, etc. O caráter visível, as imagens drásticas e os efeitos imediatos dessas manifestações patológicas induzem a protelar - equivocadamente - a atenção aos transtornos globais do desenvolvimento e aos problemas psíquicos precoces. Com uma evolução mais lenta, mas igualmente insidiosa, esses transtornos acabam provocando estragos equivalentes aos dessas patologias mais espetaculares.

Atualmente, apesar dos poucos estudos epidemiológicos, já existem evidências suficientes que demonstram uma morbidade significativa advinda dos problemas ditos emocionais, dos comportamentais, dos atrasos no desenvolvimento, das psicoses, do retardo mental e da epilepsia (Nikapota, 1991).

A incidência e a prevalência epidemiológicas dos distúrbios do desenvolvimento e dos transtornos mentais na infância são desconhecidas na maioria dos países em desenvolvimento. Um estudo multicêntrico conduzido pela Organização Mundial de Saúde - OMS (Giel e outros, 1981) aponta para uma taxa de $12 \%$ a $29 \%$ de prevalência de transtornos mentais na infância em países em desenvolvimento. Essa taxa não se mostrou diferente das de outros países. Isso revela que os transtornos mentais na infância não são irrelevantes, mas, sim, menosprezados, deixando um grande número de crianças sem assistência adequada. Outro achado deste estudo é que os profissionais da atenção primária identificam em média apenas $10 \%$ a $22 \%$ dos casos de transtornos mentais que chegam aos serviços (Ibid.). Ou seja, entre $80 \%$ e 90\% dos problemas de saúde mental infantil são perdidos (não diagnosticados) na atenção básica. Essas duas conclusões do estudo conduzidos pela OMS já apontam para a necessidade de ações que melhorem o funcionamento da atenção básica à saúde da população infantil e capacitem os profissionais dessa área para a detecção e o manejo dos transtornos mentais infantis.

No Brasil, não existem bases de dados confiáveis que possam sustentar estudos epidemiológicos e um planejamento de serviços adequado. As informações do Datasus, base de dados do Sistema Único de Saúde - SUS revelam que, de fato, os profissionais de saúde não sabem diagnosticar adequadamente os transtornos mentais específicos da infância. Observa-se uma melhora na qualidade diagnóstica em serviços universitários, que dedicam maior atenção à formação dos profissionais e à qualidade do atendimento (Assumpção Jr. e Carvalho, 1999).

Além disso, segundo Robert Kohn (apud Almeida Filho, 1985), somente um estudo no Brasil examina a prevalência dos transtornos mentais na infância. De acordo com esse estudo, 23\% das crianças, entre 5 e 14 anos (da amostra de 829 crianças de um bairro de classe média de Salvador), apresentaram algum tipo de transtorno. 
Diante dessas razões, é recomendável que os cuidados com o desenvolvimento psíquico sejam pensados como um problema de saúde pública, e sua avaliação e seu acompanhamento incorporados pelos programas de assistência materno-infantil já existentes, que assim possibilitariam oferecer atendimento "integral” à saúde da criança.

\section{A dificuldade do diagnóstico na primeira infância}

Durante a primeira infância, as manifestações comportamentais que se operam nas pequenas crianças têm um caráter acentuadamente polimorfo. Assim, uma manifestação motora é ao mesmo tempo neuro-maturativa, psicológicocognitiva, tem valor trófico-fisiológico, e também é - simultaneamente expressão emocional e significativa da relação com os outros em geral e com o meio. Dentre todos esses determinantes, a discriminação daquele predominante no momento mesmo da manifestação é algo bastante difícil. Geralmente a interpretação que atribui predominância de um tipo sobre outro depende sobretudo da formação teórica do observador. Por isso, os signos habitualmente legíveis a partir da semiologia médica, criados para as situações em que o paciente pode acrescentar a informação verbal, tornam-se confusos nas idades do infans - a criança que ainda não fala - por causa de sua polivalência. Um procedimento comum para fazer frente a essa dificuldade é reduzir essa significação múltipla a um número objetivo, eliminando qualquer consideração acerca da subjetividade.

As regurgitações (sejam por estreitamento de piloro, por estados de ansiedades na relação mãe-filho, por quadros de lactossemia diversos, etc.), as cólicas, as quedas imunológicas no sistema respiratório, os espasmos de soluço, as alterações de sono - para mencionar alguns - são quadros que tipicamente colocam os pediatras numa certa situação de perplexidade diante da evidência de um fator que escapa à leitura puramente objetiva: o fator psíquico. Diferentemente dos outros signos clássicos da semiologia médica, estes não são legíveis nem decifráveis na avaliação do signo mesmo, mas num sistema de relação que requer uma observação distante do quadro e de sua manifestação imediata.

Esse sistema de relação - que inclui a relação mãe-filho - precisa de indicadores específicos que permitam a leitura diferenciada das manifestações psíquicas do bebê, que nele são necessariamente polimorfas.

\section{A importância da detecção precoce dos problemas de desenvolvimento infantil}

Até bem pouco tempo, acreditava-se que o diagnóstico de patologias graves só podia ser estabelecido depois que a criança atingisse dois anos e meio (Crossley, 1997). Mas pesquisas recentes apontam para a possibilidade de detecção aos 18 


\section{EDITORIAL}

ano VI, n. 2, jun/ 2003

meses (Baron-Cohen e outros, 1992) e até mesmo mais cedo, aos 4 meses (Laznik, 1999).

Essa tendência de pesquisa e de tratamento tornou-se possível devido à acumulação de uma casuística e de uma experiência clínica significativas, acompanhadas de resultados alentadores. Graças a esse acúmulo de experiência, acompanhado de uma produção teórica vigorosamente acelerada nos últimos vinte anos, muitos psicanalistas puderam refinar sua escuta e rastrear, nos relatos da história clínica trazida pelos pais, sinais precoces das patologias já instaladas, sinais que não haviam sido lidos como indicadores do que viria a seguir, mas que poderiam ser, a posteriori, assim interpretados.

Diante da experiência agora acumulada, adquirem especial relevância as pesquisas que possam vir a confirmar o valor preditivo de sinais precoces de risco para o desenvolvimento infantil.

Exame das escalas de avaliação do desenvolvimento infantil mais usadas no Brasil

A maioria das escalas é de ordem descritiva, classificatória e busca medir comportamentos. As variáveis articuladas com a constituição da subjetividade aparecem pouco ou não aparecem, são excluídas ou negligenciadas.

Apresentam-se aqui algumas das escalas mais usadas no Brasil:

\section{Denver Developmental Screening Test (DDST)}

Esta escala tem como objetivo a detecção precoce de problemas ou atraso do desenvolvimento em crianças menores de seis anos. É um teste psicométrico, multifatorial, que avalia quatro grandes áreas do desenvolvimento: pessoal-social; fino motor-adaptativo; linguagem; e grosso motor. Os itens são pesquisados por meio do exame direto da criança ou em perguntas dirigidas aos pais ou cuidador.

A escala foi padronizada com boa confiabilidade. É fácil de aplicar, econômica e muito adequada para a situação de clínica pediátrica, já que não exige equipamento sofisticado ou especial. Pode ser um ótimo instrumento mesmo para pesquisa, se for levado em consideração que se destina a screening (despistagem) e nunca para um diagnóstico definitivo de problemas de desenvolvimento.

\section{Gesell}

Sem dúvida a escala mais conhecida e usada pela pediatria nas Américas. Extensa e completa, visa principalmente ao diagnóstico de problemas do 
desenvolvimento e da maturação, por meio do estudo do comportamento da criança. Para os autores, "comportamento" é um termo conveniente para todas as reações da criança, sejam elas reflexas, voluntárias, espontâneas ou aprendidas. Nessa perspectiva, o desenvolvimento é um entendido como um processo de padronização, e um padrão comportamental é simplesmente uma resposta definida do sistema neuromotor a uma situação específica. Assim, o desenvolvimento é considerado como passível de diagnóstico porque a construção do sistema de ação do bebê e da criança é, dessa perspectiva, um processo ordenado.

Para Gesell, o comportamento normal assume padrões característicos à medida que se desenvolve; conseqüentemente, o diagnóstico do desenvolvimento consiste na observação discriminativa dos padrões comportamentais e em sua avaliação pela comparação com padrões de comportamento normais, expressos em termos de idade.

\section{Bayley}

A escala Bayley de desenvolvimento infantil, em sua segunda versão, abrange o período de 1 a 42 meses. Nela constam três áreas: a motora, a mental e a comportamental. Na escala mental são incluídos itens relativos à memória, resolução de problemas, conceito de número, generalização, classificação, linguagem e habilidades sociais. Na escala motora são avaliadas a coordenação motora fina e ampla e, na escala comportamental, as atitudes e reações comportamentais frente ao teste.

Trata-se de uma escala que demanda longo tempo de aplicação, além da utilização de diversos materiais (brinquedos, pranchas, livrinhos, etc.) e um complexo treinamento por parte do aplicador. Esse treinamento não está, porém, amplamente ao alcance dos brasileiros - o Brasil conta com um número limitado de pesquisadores habilitados a utilizá-la e a realizar o treinamento de outros profissionais.

\section{Classificação diagnóstica 0-3}

Esta classificação diagnóstica propõe-se a apresentar de maneira sistematizada os transtornos psicopatológicos da criança até o terceiro ano de vida. Ela classifica modelos emocionais e comportamentais que representam diferenças significativas em relação ao desenvolvimento normal. A classificação 0-3 responde a diversos objetivos: permite a sistematização das observações clínicas; contribui para uma melhor avaliação na direção das intervenções terapêuticas e numa posterior verificação de resultados; viabiliza a utilização de uma linguagem comum aos pesquisadores além de constituir-se num modelo inicial, aberto a reformulações posteriores. 


\section{EDITORIAL}

ano $\mathrm{VI}$, n. 2, jun/ 2003

A classificação 0-3 se apresenta dividida em cinco eixos: o diagnóstico primário e a classificação de problemas da relação; afecções médicas e problemas do desenvolvimento; os fatores de estresse emocional e por fim o nível funcional do desenvolvimento emocional.

No entanto, a utilização da classificação diagnóstica 0-3 limita-se a profissionais da área de saúde mental, por se tratar de uma complexa classificação que propõe discriminações sutis entre os quadros psicopatológicos.

\section{Ficha de Avaliação do Desenvolvimento do Ministério da Saúde}

No início dos anos 1980, quando o Ministério da Saúde propôs elaborar as normas de atendimento à criança de zero a cinco anos, nas quais se incluía o Acompanhamento do Crescimento e Desenvolvimento, foi necessária a construção de uma ficha simplificada para a monitoração do desenvolvimento. O motivo era que as fichas existentes e usadas por especialistas ou pediatras eram extensas e de certa forma ainda complexas para os serviços básicos de saúde.

Um grupo de especialistas encarregou-se de elaborar uma ficha pequena, de uso simplificado, mas que ao mesmo tempo permitisse a "suspeita" de problemas no desenvolvimento psicomotor da criança. Para a elaboração da ficha optou-se por três áreas dos comportamentos - grosso motor, psicomotor e adaptativo ou social - e foram tomadas como referência as escalas comumente mais usadas, como as escalas Denver, Gesell e Mary Sheridan. (Ministério da Saúde, 1984)

\section{Avaliação crítica das escalas}

Como se pode verificar, a maior parte das escalas parte do pressuposto de que a criança pode ser avaliada em comportamentos e/ou habilidades sem relação entre si. Essas escalas, além de darem mais ênfase para o que ocorre com a criança, deixam para o segundo plano o adulto ou cuidador. Com exceção da 0-3, as demais escalas não partem de uma concepção da criança como parte de um mundo essencialmente simbólico que requer uma estrutura psíquica para organizar todas as suas funções, como a teoria e a clínica psicanalítica nos permitem pensar após tantos anos de investigação do psiquismo humano.

Desta forma, não existe um instrumento que seja de fácil aplicação e que possa verificar como essa estrutura psíquica está se constituindo, o que permitiria interrelacionar funções e habilidades a partir das quais a criança dá sentido ao mundo e não apenas verificar comportamentos resultantes de habilidades geneticamente programadas. 
À medida que essa estrutura se funda a partir da mãe ou do cuidador colocado em certa posição nos primeiros anos de vida, é essencial avaliar como se dá o estabelecimento desse laço entre mãe e bebê.

A contribuição da presente pesquisa

A partir do exposto, pode-se perceber a importância da criação de instrumentos que, construídos a partir da clínica psicanalítica, permitam a identificação dos sinais precoces de risco para o desenvolvimento. Assim, esperase, a partir desta pesquisa, converter esses indicadores em instrumento para uso diagnóstico-epidemiológico e sobretudo terapêutico, associando-o a um plano de prevenção de distúrbios na infância. Pretende-se ainda obter uma metodologia de formação de pediatras como agentes de prevenção e detecção de sinais precoces de risco para o desenvolvimento infantil.

\section{Indicadores clínicos de risco}

Para a concretização desta pesquisa, tornou-se necessária a construção de um instrumento baseado em uma fundamentação teórica, em uma consulta a experts e em dois estudos-piloto realizados previamente à proposição do presente estudo.

\section{Fundamentação teórica}

Para estabelecer os indicadores de risco para o desenvolvimento psíquico de uma criança é necessário situar de modo claro o conceito de desenvolvimento que está sendo tomado como referência. Quando se adotam indicadores por área funcional (psicomotora, perceptiva, cognitiva, social, etc.), a resultante é uma concepção fragmentadora. Especialmente no trabalho com bebês e crianças pequenas, medições por funções supostamente autônomas ocultam as formações sintomáticas complexas próprias dos momentos precoces da vida. Os pediatras em geral têm se guiado por indicadores neuropsicomotores, os quais, tomados separadamente, ou mesmo em conjunto, têm se mostrado fracamente indicativos de dificuldades propriamente psíquicas incidentes no desenvolvimento em geral. Tais sinais têm se mostrado também pouco indicativos de alterações mentais derivadas de problemas de desenvolvimento.

Dispor de aparelho perceptivo que funcione bem, ter uma evolução postural psicomotora adequada ou ter uma mãe carinhosa que brinca com ela, nenhuma 
dessas coisas, isoladamente, determina que uma criança possa evoluir psiquicamente de um modo adequado ou não.

Como já foi dito anteriormente, a perspectiva de desenvolvimento na qual se baseia a psicanálise parte do princípio de que a subjetividade é um aspecto central e organizador do desenvolvimento em todas as suas vertentes. Essa subjetividade, por sua vez, é construída pela inserção da criança na linguagem e na cultura. O que caracteriza o bebê humano é o fato de que seus instintos préformados são ressignificados por seu meio ambiente, o que o distancia do funcionamento animal em sua dimensão etológica. Nessa perspectiva, dá-se a possibilidade de uma abertura para a linguagem, que vai marcar e organizar as funções orgânicas, anatômicas, musculares, neurofisiológicas da criança, a partir do laço que ela estabelece com um outro humano, geralmente a mãe ou o cuidador.

Esse circuito é chamado de pulsional porque organiza os ritmos de satisfação e de relação do bebê com seu corpo e com o corpo do outro, entendidos aqui em uma dimensão erógena e não biológica. O ritmo do desenvolvimento vai ser então regulado pelo desejo deste outro humano - Outro, com maiúscula, para denominar aquele que está em posição de exercício dessa função de sustentar e orientar a evolução da criança.

$\mathrm{O}$ ordenamento da linguagem - desde que personalizado por um adulto desejante - é o eixo que move, organiza, interfere e configura as linhas gerais do desenvolvimento. Portanto, há uma estrutura simbólica que organiza o processo de desenvolvimento, de modo a permitir o surgimento de um sujeito. Esse surgimento depende, então, das significações que se colocam em jogo no cuidado do bebê.

Só será possível dizer que um bebê está construindo uma subjetividade a partir de certas manifestações que possam ser identificadas como respostas do sujeito, ou seja, singulares, próprias daquela criança em particular. Exemplificando, podemos dizer que uma criança autista pôde adquirir a fala por ter adquirido capacidades fono-articulatórias compatíveis com sua idade, mas pode ser que ela não esteja apta a responder como sujeito, ou seja, que ela não utilize essa fala para produzir um laço próprio, significativo e desejante com o Outro. Da mesma forma, uma criança com síndrome de Down, que apresenta dificuldades instrumentais relativas à motricidade ou à cognição impostas pela síndrome, pode ou não apresentar sinais de subjetivação independentemente de tais dificuldades instrumentais.

As atividades básicas que constituem a vida de um bebê em seus primeiros meses, tais como dormir, acordar, mamar, olhar ou defecar dependem não somente do puramente orgânico, mas também das marcas simbólicas efetuadas por seus cuidadores, primeiros agentes e transmissores dessas marcas - seus pais. Tais marcas, ao serem impressas sobre o equipamento neuroanatômico do 
bebê, colocam uma referência fundamental para o seu funcionamento. ${ }^{1}$ Por isso, trata-se de investigar o desenvolvimento da criança de modo articulado à constituição psíquica.

Para que se instale no bebê esse circuito de satisfação que o enlaça com o Outro e permite o seu desenvolvimento, a mãe precisará sustentar certas funções: - Estabelecer a demanda da criança: as primeiras reações involuntárias que um bebê apresenta ao nascer, tais como o choro, precisam ser entendidas pela mãe como um pedido que a criança dirige a ela e que a mãe se coloca em posição de responder. Isto inicialmente implica uma interpretação em que a mãe usa a linguagem, "traduz" em palavras as ações da criança e "traduz" em ações suas próprias palavras.

- Supor um sujeito: trata-se aí de uma antecipação, pois o bebê ainda não está constituído como sujeito, mas tal constituição depende justamente de que ele seja inicialmente suposto ou antecipado. É a partir dessa suposição, por exemplo, que o grito do bebê poderá ser tomado como um apelo e assim interpretado, abrindo para ele a possibilidade de, em sua emissão seguinte, já estar efetivamente marcado por uma significação de apelo.

- Alternar presença-ausência: implica que a mãe ou o cuidador não responda ao bebê apenas com presença ou apenas com ausência, mas que produza ali uma alternância, não apenas física, mas sobretudo simbólica. Para que um bebê se torne um ser desejante (o que equivale a ser autônomo e singular), é necessário que ele possa ter uma experiência de falta. Mas não uma falta qualquer meramente física, por exemplo -, mas de uma falta que possa ser falada. É por isso que, para o bebê se tornar um ser de linguagem, será necessário que as inscrições psíquicas se ordenem de modo descontínuo, alternado, e é a alternância presença-ausência oferecida pelo agente materno que poderá produzir essa descontinuidade.

- Instalação da função paterna: quando essa função se instala, a criança renuncia às satisfações imediatas que antes advinham da relação com o próprio corpo e com o corpo da mãe ou de seu cuidador. Para que a função paterna opere, é preciso que a mãe situe a lei como uma referência a um terceiro em seu laço com a criança, não fazendo desta criança um objeto que se presta unicamente à sua satisfação. É graças à ação da função paterna que uma criança poderá distanciar-se do outro materno e utilizar então a linguagem em sua função

1. Por exemplo, nas crianças que desenvolvem um quadro de hospitalismo e entram em depressão anaclítica - precisamente causada pela interrupção dos laços afetivos - a ausência dessas marcas simbólicas provoca uma desorganização neurofisiológica generalizada (ver o conceito de hospitalismo em Spitz, O primeiro ano de vida. São Paulo: Martins Fontes, 1996, p. 207. 
simbólica. Ao mesmo tempo, isso a empurra na direção de procurar novas formas de satisfação.

Esses diferentes eixos - estabelecimento da demanda, suposição de sujeito, alternância de presença-ausência e função paterna - não comparecem separadamente no decorrer do desenvolvimento, mas se entrelaçam nos cuidados que a mãe dirige à criança e também nas produções que a criança realiza, dando testemunho dos efeitos de inscrição de tais marcas. Não aparecem, então, como funções separadas ou autônomas, porém fazendo parte e orientando as funções, tanto físicas como psicológicas.

Consulta a experts e revisão bibliográfica

A instalação da subjetividade só pode ser verificada a partir dos efeitos indiretos que essa instalação determina. Assim, a leitura dessa instalação na criança apóia-se em sinais fenomênicos que, articulados em uma lógica lingüísticosimbólica, permitem supor a presença de uma subjetividade. Esses sinais irão compor os indicadores, a partir dos quais propomos investigar como está ocorrendo a constituição psíquica de modo articulado ao desenvolvimento da criança.

Segundo o conhecimento teórico-clínico já construído pela psicanálise sobre as diferentes operações psíquicas que se estabelecem na primeira infância, a partir do que entra em jogo no laço entre mãe e filho, destacaram-se observações significativas dos diferentes tempos tanto do circuito pulsional quanto da inscrição simbólica, para determinar o que seria esperado em cada período da constituição da subjetividade.

Assim, constituiu-se um grupo de experts, que recolheu, dentre essas observações significativas, aqueles indicadores já comumente utilizados no cotidiano da clínica psicanalítica com crianças e bebês e que apresentam, por sua ocorrência freqüente, uma plausibilidade clínica, relativa simplicidade de leitura, operacionalidade e transmissibilidade clínica, configurando assim o conjunto de índices cuja ausência poderá apontar para a existência de problemas de desenvolvimento.

O grupo de experts constituído elaborou também o presente projeto de pesquisa e deverá ainda encarregar-se de sua realização. Será doravante denominado GNP - Grupo Nacional de Pesquisa.

Estudo-piloto

Os estudos-piloto relativos à Pesquisa Multicêntrica de Indicadores Clínicos para a Detecção Precoce de Riscos no Desenvolvimento Infantil foram realizados nos seguintes centros: 
- São Paulo, no Centro de Saúde Samuel Pessoa, no período de 11 de junho a 13 de julho de 2001;

- Brasília, no ambulatório de pediatria do Hospital Universitário de Brasília - HUB - DF, nos períodos de 6 de agosto a 14 de setembro de 2001 e de 22 de novembro a 22 de dezembro de 2001.

Os estudos-piloto tiveram como objetivos: 1 ) verificar a confiabilidade dos indicadores; 2) avaliar o programa de capacitação dos pediatras; 3) dar as bases para a construção do protocolo do estudo multicêntrico.

Em São Paulo, o estudo-piloto foi realizado com todas as crianças da faixa etária de 0 a 18 meses que procuraram o centro de saúde no período mencionado. Já em Brasília, em função dos resultados epidemiológicos e estatísticos do piloto I, a amostra foi redefinida e se aplicaram então os protocolos apenas nas crianças atendidas no ambulatório de risco do Hospital Universitário: prematuras, filhos de mães adolescentes e que apresentavam alguma patologia orgânica.

Apresenta-se o instrumento Indicadores de Risco para o Desenvolvimento Infantil (IRDI) no anexo 1.

Construiu-se também um adendo ao protocolo, que deverá acompanhá-lo sempre, com orientação aos pediatras de como aplicá-lo e informações básicas sobre cada indicador importante para a correta observação e entendimento sobre eles. Essas instruções encontram-se no anexo 2.

\section{Concepção da pesquisa sobre o desfecho clínico}

Pode-se afirmar que o desfecho clínico da presente pesquisa coincide com as categorias psicopatológicas descritas atualmente no DSM-IV, manual editado pela Associação Americana de Psiquiatria (1994). No entanto, não seria possível utilizá-lo, tendo em vista as diferenças teórico-conceituais que nos distanciam dele. Assim, a concepção a respeito do que sejam distúrbios globais de desenvolvimento será extraída daquela em que se apóia a Classificação Diagnóstica de Saúde Mental e Transtornos de Desenvolvimento do Bebê e da Criança Pequena "Zero a Três", do Centro Nacional para Bebês, Crianças e Famílias, de Arlington, EUA.

O GNP pretende, porém, construir um novo desfecho clínico a partir da classificação diagnóstica 0-3 durante a realização da pesquisa, detalhando, precisando e ampliando a noção de Distúrbios Globais do Desenvolvimento a partir do modelo psicanalítico adotado. 
- Verificar a capacidade de predição dos indicadores clínicos de risco para distúrbios globais de desenvolvimento infantil.

- Capacitar médicos da rede pública para a identificação dos indicadores clínicos de risco para distúrbios de desenvolvimento infantil;

- Descrever o perfil epidemiológico dos usuários da rede pública de atenção à criança em relação aos indicadores clínicos de risco para distúrbios de desenvolvimento infantil;

- Verificar a associação dos indicadores clínicos de risco para distúrbios de desenvolvimento infantil com as características clínicas de crianças atendidas na rede pública;

- Selecionar indicadores de desenvolvimento psíquico para serem incluídos na ficha de acompanhamento do Desenvolvimento de Crianças de 0 a 5 anos proposta pelo Ministério da Saúde.

\section{Metodologia}

Desenho da pesquisa

Estudo de corte transversal seguido de um estudo longitudinal.

\section{Área estudada}

O estudo será desenvolvido em serviços de saúde públicos, distribuídos em dez capitais brasileiras: São Paulo, Rio de Janeiro, Curitiba, Porto Alegre, Brasília, Recife, Salvador, Belém do Pará, Fortaleza e Belo Horizonte. Tais centros foram escolhidos para garantir uma boa representatividade dos centros urbanos das diferentes regiões do país. 


\section{Amostra}

A amostra será composta por crianças nas faixas etárias de 0 a 3 meses e 29 dias; 4 a 7 meses e 29 dias; 8 a 11 meses e 29 dias; 12 a 18 meses, atendidas na clínica pediátrica de serviços públicos de saúde. Na primeira fase do estudo será selecionada uma amostra representativa de crianças em cada um dos extratos compostos pelas faixas etárias. Na segunda fase do estudo, cada uma das crianças selecionadas será acompanhada até completarem 18 meses, quando então será composta uma sub-amostra baseada na presença ou não de problemas nos IRDIs. Esta será composta de todas as crianças que apresentarem problemas até um máximo de 500, e 500 sorteadas entre aquelas que não apresentarem qualquer problema em todos os IRDIs. As crianças dessa subamostra serão acompanhadas até completarem 3 anos de idade.

O cálculo da amostra inicial foi baseado em dados do estudo-piloto realizado em dois centros (São Paulo e Brasília) e do levantamento do número de atendimentos por mês em cada serviço envolvido no estudo. O estudo-piloto mostrou prevalências baixas (variavam de $0 \%$ a 34\%) de problemas apresentados nos IRDIs em todas as faixas etárias. Considerando que este envolveu um número não representativo dos atendimentos realizados, adotamos a prevalência de $20 \%$ como referência para o cálculo da amostra.

Para a definição do número total de crianças atendidas durante um mês utilizou-se um levantamento prévio realizado em alguns dos serviços envolvidos que teve como objetivo estimar a proporção de crianças atendidas por faixa etária. Na Tabela 1 é possível observar os resultados desse levantamento em cada serviço e a estimativa para os dois serviços cujos números não obtivemos (estimativa feita a partir da média de atendimentos nos serviços, retirado o serviço do Butantã). A estimativa para o total de atendimentos por mês foi de aproximadamente 3.492 crianças.

Para a primeira fase do estudo o número necessário é de 683 crianças. Esse cálculo da amostra foi baseado nos seguintes parâmetros: prevalência esperada para o IRDI - 20\%; precisão absoluta - 3\% (indica o quanto a estimativa deve se distanciar da verdadeira proporção); nível de significância - 5\%.

Para a segunda fase do estudo o número necessário é de 878 crianças, das quais 439 deverão apresentar problemas no IRDI e 439, não. Esse cálculo da amostra foi baseado nos seguintes parâmetros: proporção de caso entre os expostos - 10\%; proporção de casos entre os não expostos - 5\%; nível de significância - 5\%; teste bicaudal. O cálculo final de 500 crianças por grupo levou em consideração o acréscimo de $14 \%$ devido as prováveis perdas ao longo do seguimento. 
Tabela 1

Número de atendimentos por mês distribuídos por serviço de saúde e faixa etária das crianças atendidas

\begin{tabular}{|c|c|c|c|c|c|c|c|c|c|}
\hline \multirow[t]{2}{*}{ Cidade } & \multirow[t]{2}{*}{$\begin{array}{l}\text { Atendimen- } \\
\text { tos por mês }\end{array}$} & \multicolumn{2}{|c|}{$\begin{array}{l}0 \text { a } 3 \\
\text { meses }\end{array}$} & \multicolumn{2}{|c|}{$\begin{array}{c}4 \text { a } 7 \\
\text { meses }\end{array}$} & \multicolumn{2}{|c|}{$\begin{array}{l}8 \text { a } 11 \\
\text { meses }\end{array}$} & \multicolumn{2}{|c|}{$\begin{array}{r}12 \text { a } 18 \\
\text { meses }\end{array}$} \\
\hline & & $\mathrm{N}$ & $\%$ & $\mathrm{~N}$ & $\%$ & $\mathrm{~N}$ & $\%$ & $\mathrm{~N}$ & $\%$ \\
\hline Brasília & 200 & $\begin{array}{l}10 \\
4\end{array}$ & $\begin{array}{l}52 \\
0\end{array}$ & 39 & $\begin{array}{l}19 \\
5\end{array}$ & 22 & $\begin{array}{l}11 \\
0\end{array}$ & 34 & $\begin{array}{l}17 \\
0\end{array}$ \\
\hline Recife & 240 & 0 & & 0 & & 0 & & 0 & \\
\hline Rio de Janeiro & 330 & 60 & $\begin{array}{l}18 \\
2\end{array}$ & 80 & $\begin{array}{l}24, \\
2\end{array}$ & 80 & $\begin{array}{l}24, \\
3\end{array}$ & 60 & $\begin{array}{l}18 \\
2\end{array}$ \\
\hline $\begin{array}{r}\text { Porto Alegre } \\
\text { (estimativa) }\end{array}$ & 234 & 0 & & 0 & & 0 & & 0 & \\
\hline Curitiba & 280 & 43 & $\begin{array}{l}15 \\
4\end{array}$ & 70 & $\begin{array}{l}25, \\
0\end{array}$ & 77 & $\begin{array}{l}27, \\
5\end{array}$ & 90 & $\begin{array}{l}32, \\
2\end{array}$ \\
\hline Belém & 205 & 60 & $\begin{array}{l}15 \\
4\end{array}$ & 40 & $\begin{array}{l}25 \\
0\end{array}$ & 85 & $\begin{array}{l}27 \\
5\end{array}$ & 20 & $\begin{array}{l}32, \\
2\end{array}$ \\
\hline $\begin{array}{l}\text { Salvador } \\
\text { (estimativa) }\end{array}$ & 234 & 0 & & 0 & & 0 & & 0 & \\
\hline $\begin{array}{l}\text { São Paulo } \\
\text { (FMUSP) }\end{array}$ & 147 & 52 & $\begin{array}{l}35 \\
4\end{array}$ & 35 & $\begin{array}{l}23 \\
8\end{array}$ & 30 & $\begin{array}{l}20 \\
4\end{array}$ & 30 & $\begin{array}{l}20 \\
4\end{array}$ \\
\hline $\begin{array}{l}\text { São Paulo } \\
\text { (Butantã) }\end{array}$ & 1.622 & $\begin{array}{l}10 \\
7 \\
\end{array}$ & 6,6 & $\begin{array}{l}41 \\
1\end{array}$ & $\begin{array}{l}25 \\
4\end{array}$ & $\begin{array}{l}41 \\
4 \\
\end{array}$ & $\begin{array}{l}25 \\
5\end{array}$ & $\begin{array}{l}69 \\
0 \\
\end{array}$ & $\begin{array}{l}42, \\
5\end{array}$ \\
\hline $\begin{array}{l}\text { Total s/ } \\
\text { (Re, Poá, Sa) }\end{array}$ & 2.784 & $\begin{array}{l}42 \\
6\end{array}$ & $\begin{array}{l}15 \\
3\end{array}$ & $\begin{array}{l}67 \\
5\end{array}$ & $\begin{array}{l}24, \\
2\end{array}$ & $\begin{array}{l}70 \\
8\end{array}$ & $\begin{array}{l}25 \\
4\end{array}$ & $\begin{array}{l}92 \\
4\end{array}$ & $\begin{array}{l}33 \\
2\end{array}$ \\
\hline Total & 3.492 & & & & & & & & \\
\hline
\end{tabular}

Embora para a primeira fase sejam necessárias apenas 683 crianças, o estudo está dimensionado para a obtenção das 500 crianças que apresentam problemas no IRDI. Para tanto, a estimativa é de que, se forem observadas 1.000 crianças, selecionadas aleatoriamente, a amostra será alcançada em aproximadamente seis meses de coleta. 


\section{Instrumento}

Será utilizado o IRDI. As crianças serão avaliadas por pediatras treinados previamente para fazer uso do IRDI durante a consulta clínica regular no período de 18 meses. Cada um dos indicadores será preenchido para a faixa atual de idade da criança e também para a faixa anterior, e podem ser pontuados como presentes, ausentes ou não verificados.

Variáveis clínicas da criança: Apgar aos 5 minutos, idade gestacional, peso ao nascer, sexo, queixa principal durante a consulta, diagnóstico clínico. Variáveis clínicas da mãe: idade, quantos partos e pré-natais. Variáveis demográficas: data de nascimento, posição na família, data da observação, acompanhante. Variáveis de seguimento: história clínica, psicodiagnóstico e diagnóstico psiquiátrico e psicanalítico após três anos de seguimento feitos por meio de exame clínico.

\section{Procedimentos}

Cada um dos serviços contará com pediatras treinados na observação da relação mãe-criança e no preenchimento do IRDI. Setenta por cento das crianças agrupadas por faixa etária serão sorteadas a partir de uma lista de inscrições no programa de puericultura dos serviços de saúde. As observações serão feitas em cada uma das crianças nas consultas regulares até a idade de 18 meses, ocasião em que terão preenchido os respectivos indicadores para a faixa etária atual e a imediatamente anterior nos casos pertencentes ao estudo transversal.

Após 18 meses, todas as crianças com algum indicador ausente e 30\% das que apresentaram todos os indicadores presentes serão contadas semestralmente durante três anos e avaliadas anualmente por meio de um exame clínico nos aspectos do desenvolvimento psicológico, de transtornos psiquiátricos e por meio de diagnóstico psicanalítico. Todas essas avaliações serão realizadas por consultores ad hoc, especialistas nessas avaliações.

\section{Análise dos dados}

A análise estatística dos dados será feita para cada indicador de forma independente. A análise de dados da primeira etapa do estudo será composta de uma descrição epidemiológica para estimar a prevalência dos IRDIs por faixa etária e sua associação com variáveis clínicas e demográficas. Após três anos de seguimento as crianças serão avaliadas para identificação de distúrbios globais de desenvolvimento e verificadas as associações com os IRDIs. 
Em cada cidade brasileira em que a pesquisa for realizada haverá um membro do GNP responsável por todas as ações a serem ali desenvolvidas.

As equipes dos centros

Em cada uma das capitais deverá ser constituída uma equipe de quatro monitores, para apoio às ações da pesquisa e de seu coordenador.

Os coordenadores deverão fazer um acompanhamento da pesquisa em seus respectivos centros, realizando reuniões mensais com sua equipe de trabalho e com a equipe de pediatras.

Os pediatras

Os pesquisadores serão os pediatras que trabalham regularmente nos centros da pesquisa, e se espera poder contar, em cada centro, com um número de pediatras pesquisadores em torno de dez.

A capacitação dos pediatras

A capacitação de pediatras será desenvolvida em um primeiro workshop com duração de 24 horas, a ser realizado em todos os 12 centros em que transcorrerá a pesquisa. Em seguida, serão realizadas outras três sessões de workshops, com uma duração de 4 horas em cada uma.

\section{Encaminhamentos}

As crianças que apresentarem sinais de risco no IRDI em mais de uma avaliação realizada, ou seja, em mais de uma faixa etária, deverão ser encaminhadas para tratamento psicoterápico, uma vez que se pode avaliar já nesse momento a presença de algum tipo de sofrimento do bebê.

Os encaminhamentos seguirão os procedimentos e avaliações usuais dos serviços de pediatria ou de saúde mental porventura presentes nos centros. As crianças deverão ser encaminhadas preferencialmente para os profissionais, serviços ou centros especializados ou de referência do Ministério da Saúde mais próximos dos centros em que foram inicialmente atendidas. Em cada centro da pesquisa, será realizado um levantamento desses locais para atendimento. 


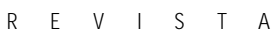

LATINOAMERICANA

DE PSICOPATOLOGIA

F U N D A MEN TA L

ano VI, n. 2, jun/2003

Os encaminhamentos e as crianças tratadas serão assinalados, e o posterior tratamento estatístico do estudo levará em conta a introdução do tratamento psicoterápico naquelas crianças que efetivamente o realizaram. Caso as crianças tratadas representem, ao final da pesquisa, um volume não desprezível da amostra, deverão ser abordadas na análise estatística como um terceiro e quarto grupos de comparação. Assim, poderão ser constituídos quatro grupos:

1) Grupo de crianças com alteração no IRDI durante a pesquisa e que não apresentou Distúrbio Global do Desenvolvimento (DGD);

2) grupo de crianças sem alteração no IRDI e que apresentou DGD ao longo do período;

3) grupo de crianças com alteração no IRDI acompanhada em tratamento e apresentando DGD;

4) grupo com alteração do indicador, acompanhada em tratamento e sem DGD.

Realização deste projeto:

1. Josenilda de Araújo Caldeira Brant (in memoriam)²

2. Grupo Nacional de Pesquisa (GNP):

Alfredo Jerusalinsky

Danielle de Brito Wanderley

Domingos Paulo Infante

Lea Salles

Leda Fischer Bernardino

M. Cecília Casagrande

Octavio de Souza

Paulina Rocha

3. Sérgio Baxter Andreoli (Estatística e Metodologia)

4. Colaboraram na realização dos estudos-piloto e na formatação do IRDI:

Equipe de São Paulo:

Adriana Grosman

Daniela Teperman

Julieta Jerusalinsky

Rachel Diaz Degenszain

Vera Ferrari Rego Barros

Wagner Ranna
Equipe de Brasília:

Adriana de Rezende Dias

Flávia Gomes Dutra

Inês Catão Henriques Ferreira

Patrícia Lima Bizerril

Sílvia Dutra Badra

2. A Dra. Josenilda de Araújo Caldeira Brant, médica pediatra, psicanalista, especialista em pediatria do desenvolvimento, mestre em saúde pública, professora aposentada da Universidade de Brasília, falecida em 12/1/02, participou ativamente da realização deste projeto, tendo sido uma das suas principais idealizadoras. 


\section{EDITORIAL}

ano $\mathrm{VI}$, n. 2, jun/ 2003

Coordenação técnico-administrativa: M. Eugênia Pesaro

Coordenação nacional: M. Cristina Kupfer

\section{Referências}

Almeida Filho, N. Epidemiologia das desordens mentais da infância no Brasil. Centro Editorial e Didático da UFBA, Salvador, Bahia, 1985.

Associação Americana de Psiquiatria. Manual de diagnóstico e estatística de distúrbios mentais (DSM-IV). 3. ed. São Paulo: Manole, 1994.

AssumpÇão JR., F.B. e Carvalho, L.N. Realidade do diagnóstico em psiquiatria infantil no Brasil. In: Jornal Brasileiro de Psiquiatria, v. 48, n. 10, p. 449-52, 1999.

Baron-Cohen et all. Can autism be detected at 18 months? The needle, the haystack, and the Снат. British Journal of Psychiatry, 161, 839-43, 1992.

Bonner, B., Milling, L. \& Walker, C.E. Denver developmental screening test. In: Jrayser, D., Sweetland, R.C. - general editors. Test Critiques, Vol. I. Test Corporation of America, 1984.

Crossley, S. A. Síndrome de Down e autismo. In: Busnel, M.C. (org.). A linguagem dos bebês, sabemos escutá-los? São Paulo: Escuta, 1997.

Gesell, A. A criança de 0 a 5 anos. 4. ed. São Paulo: Martins Fontes, 1996.

Giel, R.; Arango, M.V.; Climent, C.E.; Harding, T.W.; Ibrahim, H.H.A.; LadrigoIgnacio, L.; Murthy, R.S.; Salazar, M.C.; Wig, N.N. \& Younis, Y.O.A. Childhood mental disorders in primary health care: results of observations in four developing countries in Pediatrics, v. 68, n. 5, November/1981.

Knoblock, H. e Passamanick, B. Gesell e Amatruda: psicologia do desenvolvimento do lactente e criança pequena - Bases neuropsicológicas e controversas. São Paulo: Atheneu, 2000.

Laznik-Penot, M-C. Poderíamos pensar numa prevenção da síndrome autística? In:

Wanderley, D. Palavras em torno do berço. Salvador: Ágalma, 1997.

MinistéRIo da SAÚdE. Acompanhamento do crescimento e desenvolvimento - Normas Técnicas, Brasília, 1984.

Nikapota, A.D. Child psychiatry in developing countries. British Journal of Psychiatry, n. 158, p. 743-51, 1991.

Zero to three. Diagnostic classification: 0-3. Washington, National Center for Infants, Toddlers and Families, 1994. 